University of South Carolina

Scholar Commons

4-2007

\title{
"Wenn Vernunft volle Gewalt über das Begehrungsvermögen hätte": Über die gemeinsame Wurzel der Kantischen Imperative
}

Konstantin Pollok

University of South Carolina - Columbia, pollok@sc.edu

Follow this and additional works at: https://scholarcommons.sc.edu/phil_facpub

Part of the Philosophy Commons

\section{Publication Info}

Published in Kant-Studien, ed. Manfred Baum, Bernd Dörflinger, Heiner F. Klemme, Volume 98, Issue 1, 2007, pages 57-80.

Pollok, K. (2007). "Wenn Vernunft volle Gewalt über das Begehrungsvermögen hätte": Über die gemeinsame Wurzel der Kantischen Imperative. Kant-Studien, 98(1), 57-80.

DOI: $10.1515 /$ KANT.2007.03

(C) Kant-Studien, 2007, Walter de Gruyter

http://www.degruyter.com/view/j/kant.2007.98.issue-1/kant.2007.003/kant.2007.003.xml?format=INT

This Article is brought to you by the Philosophy, Department of at Scholar Commons. It has been accepted for inclusion in Faculty Publications by an authorized administrator of Scholar Commons. For more information, please contactdigres@mailbox.sc.edu. 


\title{
„Wenn Vernunft volle Gewalt über das Begehrungsvermögen hätte " - Über die gemeinsame Wurzel der Kantischen Imperative
}

\author{
von Konstantin Pollok, Marburg
}

I.

Kant hat mit seiner universalistischen, formalistischen und kognitivistischen Konzeption moralisch-praktischer Gründe in Gegenüberstellung zu seiner instrumentalistischen Konzeption nicht-moralisch-praktischer Gründe eine Begrifflichkeit geschaffen, die es erlaubt, handlungsleitende Überzeugungen hinsichtlich ihres Geltungsanspruchs und ihrer Verbindlichkeit zu differenzieren. Von einem ,Sollen“ spricht Kant in beiden Fällen. Nicht nur der kategorische Imperativ, der unbedingt gebietet, „ich soll niemals anders verfahren als so, daß ich auch wollen könne, meine Maxime solle ein allgemeines Gesetz werden" (GMS, AA 04: 402), sondern auch hypothetische Imperative, die bedingt gebieten, „ich soll etwas thun, darum weil ich etwas anderes will “ ${ }^{1}$, nehmen bei Kant die normative Form eines, Sollens“ an. In beiden Fällen ergehen also Forderungen an die Vernunft, doch es ist alles andere als klar, in welcher Beziehung diese Sollensformen zueinander stehen. Bestünde gar keine solche Beziehung, so träte praktische Vernunft im Plural auf - wohlgemerkt in einer einzigen Person, welcher sich der Sinn beider Imperativformen erschlossen hat. Da eine solche Form von Schizophrenie der Willensbestimmung nicht der Kantischen Vorstellung von praktischer Vernunft entspricht, stellt sich die Frage nach der Einheit von bedingtem und unbedingtem Sollen.

Die leitende Frage dieses Aufsatzes ist relativ eng umschrieben, zumindest gemessen an den Ansprüchen, die Kant selbst stellt: „,...] wenn reine Vernunft für sich praktisch sein kann und es wirklich ist, wie das Bewußtsein des moralischen Gesetzes es ausweiset, so ist es doch immer nur eine und dieselbe Vernunft, die, es sei in theoretischer oder praktischer Absicht, nach Principien a priori urtheilt [...].“ (KpV, AA 05: 121) Abgesehen von der Problematik der Protasis, die weiter unten

1 GMS, AA 04: 444; eine direkte Opposition der beiden Sollensformen findet sich in GMS, AA 04: 441: „Dies Verhältniß [zw. dem Willen und dessen Objekt; K. P.], es beruhe nun auf der Neigung, oder auf Vorstellungen der Vernunft, läßt nur hypothetische Imperativen möglich werden: ich soll etwas thun darum, weil ich etwas anderes will. Dagegen sagt der moralische, mithin kategorische Imperativ: ich soll so oder so handeln, ob ich gleich nichts anderes wollte. Z. E. jener sagt: ich soll nicht lügen, wenn ich bei Ehren bleiben will; dieser aber: ich soll nicht lügen, ob es mir gleich nicht die mindeste Schande zuzöge."

Kant-Studien 98. Jahrg., S. 57-80

DOI 10.1515/KANT.2007.003

(C) Walter de Gruyter 2007

ISSN 0022-8877 
noch $\mathrm{zu}$ thematisieren ist, scheint es mindestens prima facie fraglich $\mathrm{zu}$ sein, daß Kant den Anspruch der Apodosis, die Identität theoretischer Spontaneität und praktischer Autonomie, argumentativ eingelöst hat. Diese (schon von den frühesten Kant-Rezipienten aufgeworfene) $)^{2}$ Problematik eines einheitlichen Begriffs der Vernunft, also die Einheit epistemischer und praktischer Normativität, soll im folgenden jedoch ausgeklammert bleiben. Stattdessen soll die Einheit allein der praktischen Vernunft thematisiert werden. Ist „praktische Vernunft“ gemäß der Kantischen Konzeption ein Gattungsbegriff, der verschiedene Arten unter sich befaßt? In welchem Verhältnis steht diese praktische Vernunft zu den unterschiedlichen Formen des Sollens?

Ich werde zunächst auf die hierfür einschlägige Differenz zwischen den Kantischen Imperativen eingehen, wobei sich herausstellen wird, daß hypothetische Imperative einen leichteren Zugang zum Begriff praktischer Vernunft ermöglichen als der kategorische Imperativ, gleichwohl die Normativität jener mit erheblichen Problemen behaftet ist (II). Mit der Erläuterung und Beantwortung der Frage nach der normativen Kraft instrumenteller Rationalität wird zugleich die systematische Verknüpfung der hypothetischen Imperative mit dem kategorischen Imperativ sichtbar. ${ }^{3}$ Die Entdeckung der gemeinsamen Wurzel der Kantischen Imperative ermöglicht schließlich eine Bewertung der Begriffe von Heteronomie und Autonomie bezüglich ihrer Relevanz für die Kantische Vorstellung von der Bestimmung des Menschen. Meine Frage ist hier jedoch nicht, ob beispielsweise Tiere oder Heilige klug oder gar moralisch handeln können, sondern vielmehr welche Voraussetzun-

2 Vgl. dazu beispielsweise Carl Leonhard Reinholds Forderung nach einer „gemeinschaftlichen Fundamentalwissenschaft" (Reinhold 1791, 127; vgl. auch ebd. 71, 75f. und 107).

3 Damit kann eine einzige Quelle normativer Kraft ausgemacht werden, aus der sich in der Kantischen Konzeption alle Imperative, auch der kategorische, speisen. Ich halte es dabei für sinnvoll, von einer Quelle der Normativität nur in sensu stricto als der Bedingung der Möglichkeit verschiedener Formen praktischer Normativität zu sprechen. Es geht mir also nicht um diese verschiedenen Formen, beispielsweise die Bitte, den Aufruf, die Vorschrift oder die Nötigung, sondern vielmehr um Kants Konzeption eines Wesens, für welches diese Formen praktischer Normativität einschlägig sind, ja, welches seinerseits durch diese Normativität wesentlich bestimmt ist. Zunächst wird auch von Christine Korsgaard kein Plural angesprochen (der ihrem Buch The Sources of Normativity den Titel gegeben hat): „Concepts like knowledge, beauty, and meaning, as well as virtue and justice, all have a normative dimension, for they tell us what to think, what to like, what to say, what to do, and what to be. And it is the force of these normative claims - the right of these concepts to give laws to us - that we want to understand." (Korsgaard 1996, 9) Im nachfolgenden wird jedoch die entsprechende Diversifizierung der Normativität von „obligation, rightness, goodness, meaning, knowledge, beauty, and virtue“ klar: „Obligation, the most obtrusively normative of these concepts, seems sternly to command; while beauty only to attract and meaning perhaps to suggest." (Ebd. 20-21) Korsgaard ist schließlich jedoch entgegen dieser Diversifikation von Normativität (und damit auch in gewisser Hinsicht dem Buchtitel zuwider) der Auffassung, daß „a unified account of normativity“ (ebd.) möglich ist; ein derart umfassendes Konzept von Normativität innerhalb Kants Philosophie, das also die bindende Kraft von Imperativen ebenso umfassen müßte wie die von Erfahrungs- oder gar Geschmacksurteilen, ist nicht Gegenstand vorliegender Untersuchung. 
gen erfüllt sein müssen, damit eine Aufforderung zu klugem oder gar moralischem Handeln überhaupt sinnvoll ist. Wir kennen - so Kants Antwort - außer dem Menschen kein Wesen, bei dem dies zuträfe (III). ${ }^{4}$

II.

Die aus der gegenwärtigen Ethik-Debatte geläufige Differenzierung von instrumenteller, prudentieller und moralischer Rationalität spiegelt in etwa die Kantische Unterscheidung von hypothetischen Imperativen der Geschicklichkeit bzw. der Klugheit sowie dem kategorischen Imperativ der Moralität wieder. $^{5}$

Auf den ersten Blick scheint die Sollens-Geltung des kategorischen Imperativs mit weniger Problemen behaftet zu sein als die der hypothetischen Imperative. Kants "Grundgesetz der reinen praktischen Vernunft“ - „Handle so, daß die Maxime deines Willens jederzeit zugleich als Princip einer allgemeinen Gesetzgebung gelten könne“ (KpV, AA 05: 30) - läßt aufgrund seines Formalismus', seiner Inhaltsleere, nicht nur keinen Platz für Abwägungen von Interessen, seien sie egoistischer oder altruistischer Art (vgl. GMS, AA 04: 431). Kant entbindet vielmehr durch die unbedingte Verbindlichkeit dieses Imperativs diejenige Person, die aus Achtung vor diesem Sittengesetz handelt, von jeder weiteren Pflicht, die entsprechende Handlung zu rechtfertigen. ${ }^{6}$ Wer aus Achtung vor dem Sittengesetz so handelt, daß die Maxime seiner Handlung (als deren hinreichende Begründung) konfliktfrei auch für alle anderen Personen gelten kann, handelt richtig bzw. sittlich. Das Sittengesetz, wie Kant es für endliche Wesen als kategorischen Imperativ formuliert hat, läßt keinen Raum möglicher Überlegung, es ist weder Diskussionsvorschlag noch Verhandlungsbasis und - zumindest nach Kant - selbst „für den gemeinsten Verstand ganz leicht und ohne Bedenken einzusehen “ (KpV, AA 05:36), weil es im Gegensatz zu Naturgeset-

4 Wenn im folgenden vom Menschen, von menschlicher Rationalität und deren Normativität, die Rede ist, so wird damit im Sinne der Religionsschrift (1793) ein Begriff angesprochen, der die Anlagen zur Tierheit, zur Menschheit und zur Persönlichkeit umfaßt (vgl. RGV, AA 06: 26-28); vgl. dazu auch Patrick Kain (2003). Transzendentalphilosophisch ist menschliche Rationalität nach Kant durch Sinnlichkeit und Verstand/Vernunft definiert; vgl. z. B. KrV, A 15, 51, 68, 77, 277-278, 546-547, B 308-311.

5 Vgl. zu dieser Differenzierung beispielsweise Korsgaard 1997, 215-217; eine hinsichtlich der Hierarchisierung analoge und ebenfalls an Kant angelehnte Differenzierung der „Konstellation von Vernunft und Willen in pragmatischen, ethischen und moralischen Diskursen“ nimmt mit diskurstheoretischer Orientierung Jürgen Habermas $(1991,101)$ vor; vgl. dazu auch Rainer Forst 1999, 169-174. Vgl. auch Clemens Schwaiger (1999) zum historischen Vorlauf dieser „drei Grundtypen menschlicher Praxis“ in der Philosophie Kants bis zur Grundlegung.

6 Daß Kant in der Grundlegung die Geltung des kategorischen Imperativs hinsichtlich innerer/äußerer sowie engerer/weiterer bzw. vollkommener/unvollkommener Pflichten differenziert, tangiert nicht die prinzipielle Geltung des kategorischen Imperativs, sondern betrifft nur die umstandsspezifische „Einteilung der Pflichten“ (GMS, AA 04: 422) und damit die Anwendungskontexte des uneingeschränkt geltenden, d.h. kategorischen Imperativs. 
zen nicht erschlossen und erkannt, sondern nur anerkannt (oder mißachtet) wird. Die Willensnötigung des kategorischen Imperativs kommt ungefragt und fraglos auf uns zu, sie trifft uns als „Factum der reinen Vernunft“ (KpV, AA 05: 31) - ob wir wollen oder nicht. Nur seine Anerkennung respektive Mißachtung erfordert einen Akt vernünftiger Freiheit, den Kant als Autonomie bezeichnet.7

Anders verhält es sich dagegen mit den hypothetischen Imperativen. Sie „stellen die praktische Nothwendigkeit einer möglichen Handlung als Mittel zu etwas anderem, was man will (oder doch möglich ist, daß man es wolle), zu gelangen vor" (GMS, AA 04: 414). Diese Imperative sind insofern hypothetisch, als die in ihnen ausgedrückte Notwendigkeit einer Handlung bedingt ist durch die Vorgabe eines bestimmten Zwecks. ${ }^{8}$ Zwar verfolgt auch der im engeren Sinne moralisch handelnde Mensch einen Zweck; wesentlich zweck- oder absichtsloses Handeln wäre bloßes Verhalten. Doch hängt bei ihm die praktische Notwendigkeit der Handlung gerade nicht von diesem Zweck ab (vgl. KpV, AA 05: 34). Während das Gebot, Klavier zu üben, nur für diejenige Person gilt, die die Fähigkeit erlangen will, Klavier spielen zu können, gilt beispielsweise das Lügenverbot und mit ihm alle im engeren Sinne moralisch relevanten Gebote und Verbote zweckinvariant; zumindest besagt dies die Kantische Konzeption. ${ }^{9}$

Im Gegensatz zum kategorischen Imperativ gibt Kant von den hypothetischen Imperativen keine allgemeingültige Formel an. Dieter Schönecker und Allen Wood haben jedoch aus den Kantischen Beispielen die folgende, in meinen Augen sehr hilfreiche Formel extrahiert:

Wenn [...] (i) zwischen dem Zweck p und dem Mittel q ein Verhältnis der Art besteht, daß nur die Ergreifung von q die Realisation von p ermöglicht, (ii) man q ergreifen kann und (iii) man $\mathrm{p}$ will, so soll man q ergreifen ${ }^{10}$.

7 Vgl. zum ,Factum der reinen Vernunft' bereits Dieter Henrich (1960), sowie Marcus Willaschek (1992, 174-193, 330-333), Klaus Steigleder (2002, 96-108) und Rainer Forst (2004, 188-197).

8 Nach Günter Patzig (1966, 101-111) lenkt auch Konrad Cramer in diesem Zusammenhang die Aufmerksamkeit von der sprachlichen Form auf materiale Überlegungen: „So hängt die Entscheidung darüber, ob ein ,kategorisch“ oder ,hypothetisch' formulierter Imperativ ,kategorisch' oder ,hypothetisch', ,unbedingt' oder ,bedingt' gebietet, nicht von sprachlichen Erwägungen, sondern von sachlichen Unterscheidungen und deren Begründbarkeit ab. Imperative wie ,Versprich nicht lügenhaft' im Unterschied zu ,Zieh dich warm an' und, Wenn du dich nicht um deinen Kredit bringen willst, so halte deine Versprechen' im Unterschied $\mathrm{zu}$,Wenn du ein Versprechen abgegeben hast, so halte es' machen hierauf aufmerksam.“ (Cramer 1972, 160) Vgl. dazu auch Bernd Ludwig (1999, 107, 112).

9 In der GMS, AA 04: 444, heißt es lapidar: „moralisch, d. i. kategorisch“. - Die Zweckinvarianz der Geltung, von der hier die Rede ist, widerspricht nicht der oben erwähnten umstandsspezifischen „Einteilung der Pflichten“ (s.o. Fn. 6) und ist daher nicht zu verwechseln mit einer Situationsinvarianz; im Beispiel: das Lügenverbot gilt immer, d.h. unabhängig vom Zweck der Lüge, aber es kommt natürlich nur in bestimmten interpersonalen und illokutionssensiblen Kontexten zur Anwendung.

10 Schönecker/Wood 2002, 111; vgl. bereits Wood 1999, XXII. Wollte man diesen Satz grammatikalisch in eine imperative Form bringen, hätte er etwa folgende Form: „wenn du den Zweck p willst und das dazu notwendige Mittel q ergreifen kannst, so ergreife q!“-Daß die 
Unter den Voraussetzungen also, daß man erstens einen bestimmten Zweck verfolgt (vgl. GMS, AA 04: 441, 444) und zweitens die zu dessen Realisierung nötigen Mittel ergreifen kann (vgl. GMS, AA 04: 417), soll man diese Mittel ergreifen. Dieses soll ist dasjenige, was im folgenden auf dem Spiel steht.

Kant unterscheidet zwar innerhalb der hypothetischen Imperative noch assertorisch-pragmatische der Klugheit von problematisch-technischen der Geschicklichkeit (vgl. GMS, AA 04: 414-417). Doch ist im gegenwärtigen Kontext statt dieser Differenzierung eine Gemeinsamkeit zwischen diesen beiden möglichen Formen hypothetischer Imperative von größerer Bedeutung: Im Gegensatz zum kategorischen Imperativ, der ein „synthetisch-praktischer Satz a priori“ (GMS, AA 04: 420) ist, bezeichnet Kant sowohl die assertorischen Imperative der Klugheit als auch die problematischen Imperative der Geschicklichkeit als „analytisch-praktische“ Sätze (vgl. GMS, AA 04: 417, 419). Praktisch sind diese Sätze, insofern sie die Vorschrift einer Handlung beinhalten, analytisch sind sie, insofern sie bloß die notwendigen Mittel zu einem vorausgesetzten Zweck betreffen. Die geforderte Handlung ist also aus dem Zweck ableitbar. Dies scheint eine klare Bestimmung zu sein und Kant stellt mit Bezug auf hypothetische Imperative und in Absetzung vom kategorischen Imperativ lapidar fest: „Es ist also in Ansehung der Möglichkeit eines solchen [sc. hypothetischen; K. P.] Imperativs auch keine Schwierigkeit."11 Doch scheint dies noch keineswegs ausgemacht, wie man bei näherer Betrachtung der einschlägigen Begründung feststellen muß.

Die Schwierigkeit „in Ansehung der Möglichkeit eines solchen Imperativs“ richtet sich zwar nicht auf das praktisch-analytische Verhältnis zwischen dem Zweck und den notwendigen Mitteln - wer einen Zweck will, muß die zu dessen Erreichen notwendigen Mittel ergreifen, sonst erreicht er seinen Zweck eben nicht. Aber mit der Charakterisierung eines Satzes als analytisch scheint seine imperative Form un-

situationsbedingte Einsetzung für q und p eine Kontingenz auf diesen hypothetischen Imperativ überträgt, stellt meines Erachtens kein Hindernis für eine abstrakte Formulierung mit Variablen dar (vgl. GMS, AA 04: 420). Die Schönecker/Wood-Formel unterscheidet sich in gewisser Hinsicht von derjenigen Thomas E. Hills: „If a person wills an end and certain means are necessary to achieve that end and are within his power, then he ought to will those means. “ (Hill 1973, 429) Die von mir vorgenommene Hervorhebung in diesem Zitat zeigt bereits die Differenz zwischen dieser Abstraktion und der vorstehenden, die von Kant (vgl. GMS, AA 04: 444) selbst nahegelegt wird. Von der Unmöglichkeit einer allgemeinen Formel hypothetischer Imperative geht dagegen Ludwig $(1999,107)$ aus, obwohl sich offenkundig selbst nach Ludwigs Ausführungen eine allgemeine Formel nahelegt: „Wer handelt, ist genötigt, sich zwischen Einsatz des als notwendig erkannten Mittels [M] und Verzicht auf die Realisierung des Zwecks [Z] zu entscheiden.“ (B. Ludwig 1999, 121, Anm. 40)

11 GMS, AA 04: 419; Kant handelt an dieser Stelle eigentlich nur vom prudentiellen Imperativ, setzt diesen aber gleichzeitig auf dieselbe Stufe wie den instrumentellen. Steigleder 2002, 40-45, arbeitet in einer instruktiven und textnahen Analyse eine mögliche Relation zwischen „technischen Imperativen“ und „Ratschlägen der Klugheit“ heraus, wobei er deren hypothetischen (problematischen) respektive assertorischen Charakter als entscheidende Interpretamente behandelt. Vgl. zu Steigleders Argumentation für hypothetische Imperative im Sinne einer Rationalitätsforderung, ebd., 32-34. 
vereinbar zu sein. Und diese imperative Form ist - jenseits rein formalsprachlicher Überlegungen - dasjenige, was die Frage nach der Möglichkeit jeglicher Imperative ausmacht. Kant betont nämlich: „Diese Frage verlangt nicht zu wissen, wie die Vollziehung der Handlung, welche der Imperativ gebietet, sondern wie bloß die Nöthigung des Willens, die der Imperativ in der Aufgabe ausdrückt, gedacht werden könne." 12 Er stellt diese Frage nun nicht - wie man vielleicht erwarten könnte ${ }^{13}$ nur für den kategorischen Imperativ, sondern eben explizit auch für die hypothetischen Imperative. Er fragt nach der Möglichkeit der Willensnötigung durch einen solchen hypothetischen Imperativ. Doch seine Antwort scheint auf den ersten Blick verblüffend, denn er gibt als Begründung des Sollens einen deskriptiven Satz ${ }^{14}$ an, den er selbst wiederum als analytisch charakterisiert:

Wer den Zweck will, will (so fern die Vernunft auf seine Handlungen entscheidenden Einfluß hat) auch das dazu unentbehrlich nothwendige Mittel, das in seiner Gewalt ist. Dieser Satz ist, was das Wollen betrifft, analytisch; denn in dem Wollen eines Objects als meiner Wirkung wird schon meine Causalität als handelnde Ursache, d. i. der Gebrauch der Mittel, gedacht, und der Imperativ zieht den Begriff nothwendiger Handlungen zu diesem Zwecke schon aus dem Begriff eines Wollens dieses Zwecks heraus [...]. ${ }^{15}$

Die Analytizität der hypothetischen Imperative stammt also aus einem deskriptiven Satz über das menschliche Wollen - wohlgemerkt über das Wollen, denn von einem Sollen ist hier noch keine Rede. ${ }^{16}$

Kant wiederholt diese Behauptung einer volitiven analytischen Zweck-Mittel-Relation im selben Abschnitt weitere Male ${ }^{17}$, wodurch die Lücke zwischen der Analytizität dieser Relation und der behaupteten Analytizität des Imperativs nur umso deutlicher sichtbar wird. Damit verschärft sich auch die entsprechende Frage: Woher stammt die normative Kraft, die der hypothetische Imperativ im Gegensatz zu dem genannten analytischen Zweck-Mittel-Prinzip besitzt? Oder nochmals konkreter formuliert: welche normativen Ressourcen werden beansprucht, damit aus einer

12 GMS, AA 04: 417 (Hervorhebung: K.P.).

13 Vgl. dazu in der GMS, AA 04: 419, 454, die Vorarbeit zu den Prolegomena, ScheffnerNachlaß, HN, AA 23: 65, sowie meine Einleitung zur Neu-Edition von Kants Prolegomena (Hamburg 2001), XI-XII.

$14 \mathrm{Daß}$ man hier auch in Kants Sinne von einem Satz, nicht nur von einem Urteil sprechen kann, bestätigen Aussagen, die in der Jäsche-Logik dokumentiert sind: „Im Urtheile wird das Verhältniß verschiedener Vorstellungen zur Einheit des Bewußtseins bloß als problematisch gedacht, in einem Satze hingegen als assertorisch. Ein problematischer Satz ist eine contradictio in adjecto. Ehe ich einen Satz habe, muß ich doch erst urtheilen; und ich urtheile über vieles, was ich nicht ausmache, welches ich aber thun muß, sobald ich ein Urtheil als Satz bestimme. “ (Log, AA 09: 109)

15 GMS, AA 04: 417. - Der letzte Satz dieses Zitats gilt mutatis mutandis auch für das Sittengesetz. Auch das Sittengesetz ist ein analytischer Satz - allerdings nur für einen (problematischen) „heiligen Willen “; ich werde darauf in Abschnitt III zurückkommen.

16 Vgl. dazu bereits Cramer 1972, 162-163.

17 Zwei weitere Formulierungen finden sich nur wenige Zeilen nach der bereits angeführten in eben diesem „Zweiten Abschnitt“ der Grundlegung (AA 04, 417-418); vgl. dazu auch Schönecker/Wood 2002, 116-122. 
handlungsbezogenen Zweck-Mittel-Relation ein Aufruf, eine Vorschrift, ein Sollen der einschlägigen Handlung folgen kann? Daß diese Frage sinnvoll ist und Kant ausdrücklich das Zweck-Mittel-Prinzip nicht mit dem Imperativ identifiziert wissen wollte, wird aus zahlreichen Bemerkungen klar ersichtlich, die das Zweck-MittelPrinzip in eine Forderungsmodalität bringen: „wenn oder weil man dieses Object will, soll man so oder so handeln“, und wenige Zeilen später in derselben Grundlegung der oben bereits zitierte Satz: „ich soll etwas thun, darum weil ich etwas anderes will [...]." 18 Auch hypothetische Imperative ,gebieten" also.

Das Interesse an der normativen Kraft analytisch-praktischer Sätze und damit an der Umwandlung des Zweck-Mittel-Prinzips in einen hypothetischen Imperativ läßt sich damit weiter spezifizieren. Was ist die Differenz zwischen dem analytischen Urteil:

Wer den Zweck will, will (so fern die Vernunft auf seine Handlungen entscheidenden Einfluß hat) auch das dazu unentbehrlich nothwendige Mittel, das in seiner Gewalt ist. (GMS, AA 04: 417)

auf der einen Seite und dem hypothetischen Imperativ (in einer allgemeinen Formulierung) auf der anderen:

wenn du den Zweck p willst und das dazu notwendige Mittel q ergreifen kannst, so ergreife q!

Die entscheidende Differenz bezieht sich meines Erachtens auf die Rationalitätsklausel innerhalb des Zweck-Mittel-Prinzips. Diese Rationalitätsklausel, der Klammerausdruck in dem analytischen Zweck-Mittel-Prinzip, ist verantwortlich für die Umwandlung des analytischen Urteils in einen Imperativ. ${ }^{19}$ Wenn man sich die eben

18 GMS, AA 04: 444; vgl. auch GMS, AA 04: 441 und in der KpV: „Die Imperativen selber aber, wenn sie bedingt sind, d. i. nicht den Willen schlechthin als Willen, sondern nur in Ansehung einer begehrten Wirkung bestimmen, d. i. hypothetische Imperativen sind, sind [...] praktische Vorschriften [...]" (KpV AA 05: 20; Hervorhebung: K.P.). Vgl. dazu auch MP Collins, AA 27, 245-247, sowie MP Mrongovius, AA 27, 1397-1400. Im Anschluß an Cramer $(1972,212)$ hat Roswitha Staege darauf hingewiesen, daß das Zweck-Mittel-Prinzip und mit ihm der hypothetische Imperativ stets in der ersten Person Singular formulierbar sein muß: „Da der mit einem Token des Satzes ,Du willst Z“ Angeredete den Sinn dieses Satzes durch ein Token des Satzes, Ich will Z‘ auffasst, wird der mit einem hypothetischen Imperativ Angeredete zum Adressaten des Imperativs, wenn er den Satz ,Ich will Z“ behauptet. Der Imperativ richtet sich nicht an den, der Z will, sondern an den, der sich das Prädikat ,will Z‘ zuschreibt." (Staege 2002, 54) Kants Texte fordern diese restriktive Lesart zwar nicht explizit, stehen ihr aber, wie die eben zitierte Passage in GMS, AA 04: 444 zeigt, auch nicht im Weg.

19 John Broome hat hinsichtlich der Rationalitätsklausel darauf aufmerksam gemacht, daß die Mittel auf solche restringiert sind, die von der handelnden Person als notwendig gewußt sind: „Rationality requires of $N$ that, if $N$ intends that $e$, and if $N$ believes that $e$ will not be so unless $m$ is so, and if $N$ believes $m$ will not be so unless she intends that $m$, then $N$ intends that $m$. [...] This is a wide principle of instrumental rationality. It incorporates a rendering of Kant's remark: Who wills the end, wills (so far as reason has a decisive influence on his actions) also the means which are indispensably necessary and in his power [...]. Kant made a slight error; he should have said ,[...] the means he believes to be indispensable necessary $[\ldots]^{6}$, and that is corrected in my formula. More importantly, my formula does not apply 
gegenübergestellten Sätze ansieht, so bemerkt man, daß der Klammerausdruck „(so fern die Vernunft auf seine Handlungen entscheidenden Einfluß hat)“ dasjenige ist, was sich allein im Zweck-Mittel-Prinzip und nicht im hypothetischen Imperativ findet. Die anderen Bestimmungen des Zweck-Mittel-Prinzips - die beiden Voraussetzungen des gegebenen Zwecks und der Beherrschung erforderlicher Mittel - gehen unverändert in die imperative Formulierung ein. Die Rationalitätsklausel enthält somit den Grund für den Übergang vom analytischen Zweck-Mittel-Prinzip zu der Nötigung des Willens, angesichts eines gegebenen Zwecks die für dessen Realisierung erforderlichen Mittel zu ergreifen. In dieser Rationalitätsklausel befindet sich demnach die Quelle der Normativität hypothetischer Imperative. Ihr Aufgehen im Imperativ läßt sich präskriptiv und allgemein so umformulieren:

gestatte der Vernunft entscheidenden Einfluß auf deine Handlung!

Diese Aufforderung liegt systematisch eine Ebene unter den hypothetischen Imperativen. Erst die Verknüpfung dieser Aufforderung mit der Zweck-Mittel-Relation (nun ohne die Rationalitätsklausel) ergibt einen hypothetischen Imperativ, d.h. eine Aufforderung, das Zweck-Mittel-Prinzip zu realisieren. Die entsprechende Reformulierung des hypothetischen Imperativs lautet also (aus der Perspektive einer dritten Person):

Wer (i) imstande ist, willensbezogene Zweck-Mittel-Relationen zu erkennen,

(ii) ein Gebot erlassen und

(iii) dieses auch selbst befolgen kann,

gebietet sich, wenn sie oder er sich einen Zweck gesetzt hat, ipso facto den Einsatz der entsprechenden Mittel. In der Kantischen Formulierung des Zweck-Mittel-Prinzips zielt die Rationalitätsklausel auf die Realisierung oder Umsetzung von (i), (ii) und (iii).

Jean Hampton hat in diesem Zusammenhang zu Recht darauf hingewiesen, daß die Normativität hypothetischer Imperative nicht auf die in dem Ziel enthaltenen Präferenzen reduziert werden kann: „[...] a careful look at his account of the force of hypothetical imperatives shows he [sc. Kant; K.P.] believes that we are motivated

only to means you believe are indispensably necessary. If it did, it would rarely have much application. You will rarely believe that a particular means is indispensably necessary to an end you intend; you will nearly always recognize there is more than one way to achieve your end. If you intend to get milk, no doubt you believe you could buy some from a shop, but you should also recognize that you could break into your neighbour's house and steal some. This is such a bad means you will give it no attention. Nevertheless, you do not believe that buying milk from a shop is an indispensably necessary means to getting some. So Kant's formula does not apply to you. On the other hand, you probably do believe you will not get milk unless you buy it from a shop. Therefore my formula probably does apply to you. [...] Because Kant imposed the condition that (you believe - he should have said) the means should be in your power, he could have dispensed with the condition that (you believe - he should have said) it is indispensably necessary. That is what I have done." (Broome, MS 2005, Kap. 4 „Requirements of rationality“) Auf die Notwendigkeit der Ergreifung bestimmter Mittel werde ich im folgenden nicht weiter eingehen; die Erkenntnis adäquater, hinreichender oder notwendiger Mittel spielt für meine Diskussion keine zentrale Rolle. 
to follow them not by the desires assumed by such imperatives but by reason - that is, by the instrumental (and not the moral) component of reason. " 20 Und hinsichtlich des Charakters dieser Autorität der Vernunft fährt sie fort: „A hypothetical imperative is a norm that generates a reason for an agent to pursue an instrumentally efficacious action, whose authority over the agent is objective (she must follow this directive, or else be condemned as irrational). When a rational agent acts on a hypothetical imperative, she is not motivated to do so by the desire assumed in the imperative, but by the authority of the imperative [...], so that the imperative is motivationally efficacious by virtue of its authority." 21 Nicht der Zweck nötigt die handelnde Person zur Ergreifung entsprechender Mittel. Vielmehr bezieht sich die Normativität hypothetischer Imperative auf die Kohärenz der Zweck-Mittel-Relation insgesamt, welche dadurch eingelöst werden kann, daß entweder die Mittel ergriffen werden oder der Zweck fallengelassen wird. Dieses Verhältnis von Normativität und Vernunft ist von fundamentaler Bedeutung, denn wie Hampton herausgestrichen hat, wohnt nach Kant Neigungen als solchen keine - auch nicht instrumentelle - Normativität inne. Der, entscheidende Einfluß der Vernunft' geht darauf, dem praktischen Konditional insgesamt gerecht zu werden. Zwecke vererben nicht schlichtweg ihre Normativität an die entsprechenden Mittel. Wäre dies der Fall, könnten für sich betrachtet irrationale Handlungen plötzlich Rationalität erlangen; Michael Bratman hat diese Problematik unter dem Titel bootstrapping diskutiert. ${ }^{22}$

Mit dem Gebot der Ergreifung der für den angestrebten Zweck als notwendig erkannten Mittel ist natürlich ein entsprechendes Handeln noch nicht garantiert, das Phänomen der Willensschwäche also nicht ausgeschlossen; doch sowohl die Analytizität hypothetischer Imperative, die ,Ableitbarkeit' der Mittel aus dem Zweck, als auch die Autorität hypothetischer Imperative, ihr Rekurs auf die Handlungseffektivität der Vernunft in Übereinstimmung mit unseren kontingenten Zwecken, sind damit erklärt. ${ }^{23}$

20 Hampton 1998, 131.

21 Hampton 1998, 133-134.

22 Vgl. Bratman 1987, 24-27, 42-46.

23 Vgl. dazu auch Cramer 1972, 189: „,Vernünftigkeit‘ des empirischen Willens bedeutet, daß die Vorstellung eines Objekts und einer empirischen Regel, die es als Wirkung zu bestimmen erlaubt, ,objektiver Bestimmungsgrund“ ist.“ Vgl. zur obigen Analyse auch Schönecker/ Wood 2002, 120: „Es gehört [...] zur Bedeutung des Aktes, sich einen Zweck zu setzen, daß man sich damit selbst gebietet, auch die entsprechenden Mittel zu wollen. Das heißt nicht, daß man die Mittel will, wenn man einen Zweck will; aber es heißt, daß man die Mittel wollen soll. Der hypothetische Imperativ ist also in dem Sinne analytisch, daß ein normatives Prinzip - nämlich der hypothetische Imperativ selbst - mit der Aktivität des Zweckesetzens verbunden ist. "Auch Schönecker/Wood gehen also davon aus, daß nicht die in den Zwekken enthaltenen Präferenzen selbst, sondern ein eigenes, Gebieten' die Quelle der Normativität darstellt. Vgl. aber zu meiner Isolation des aus der Rationalitätsklausel generierten normativen Prinzips und damit im Gegensatz zu Schönecker/Wood auch die Rekonstruktion Gerhard Seels, der meiner Basisformel praktischer Vorschriften („gestatte der Vernunft entscheidenden Einfluß auf deine Handlung!“) ähnlich im Sinne Kants ein Prinzip „S 14“ aufstellt: „Endliche vernünftige Wesen sollen sich bei ihrer Willensbildung von der Vernunft 
Aufgabe hypothetischer Imperative ist es demnach, zu verhindern, daß ein aufgrund eines gesetzten Zwecks bestehender Wille zum bloßen Wunsch degradiert wird. Denn im Gegensatz zu letzterem hatte Kant diesen Willen charakterisiert „als die Aufbietung aller Mittel, so weit sie in unserer Gewalt sind“ (GMS, AA 04: 394). Wird diese Aufbietung aller notwendigen und uns zugänglichen Mittel nicht geleistet und gleichzeitig am Zweck festgehalten, so handelt es sich um das Phänomen der Willensschwäche. Gegen eine solche mögliche Willensschwäche wendet sich gerade die Autorität der hypothetischen Imperative.

\section{Exkurs}

\section{a) Herbert J. Paton}

Gemäß der vorstehenden Interpretation von Kants Konzeption hypothetischer Imperative ist die Möglichkeit des Menschen, widervernünftig zu handeln, indem er die zu einem selbstgesetzten Zweck bedingten Mittel nicht ergreift und damit den Willen zum bloßen Wunsch degradiert, mitverantwortlich für die Möglichkeit hypothetischer Imperative. In gewisser Hinsicht steht diese Interpretation damit derjenigen Patons nahe, insofern dieser in der Heteronomie des Menschen das theoretische Erfordernis hypothetischer Imperative sieht.

Auch Paton marginalisiert die assertorisch-pragmatischen Imperative der Klugheit mit Bezug auf die Analytizität hypothetischer Imperative und diskutiert letztere im Kontext problematisch-technischer Imperative der Geschicklichkeit. Patons Ausgangspunkt ist der Satz

,to will the end is to will the means' (Paton 1947, 124),

von dem, so darf man interpolieren, kein Weg zu einem Imperativ, welchem auch immer, führt. Kant erweitert nach Paton daher diesen Satz zu dem oben diskutierten Zweck-Mittel-Prinzip:

,Any rational agent who wills the end will necessarily - so far as reason has a decisive influence over his actions - will the means which are in his power.

bestimmen lassen.“ (Seel 1989, 168) Er fährt dazu fort: „,...] mit Hilfe von S 14 läßt sich sowohl der kategorische als auch der hypothetische Imperativ begründen. Dies berechtigt uns zu der Annahme, daß Kant dieses Prinzip akzeptiert hätte. [...] Über die Frage, warum Kant nicht selbst auf ein solches oberstes Prinzip der praktischen Vernunft verfallen ist, läßt sich nur spekulieren. Vermutlich hängt das mit der bei ihm vorherrschenden Tendenz zusammen, die beiden Bereiche der praktischen Vernunft scharf von einander zu trennen und die technische Vernunft gegenüber der reinen praktischen Vernunft abzuwerten. “ (Ebd. 169) Auf die der Rationalitätsklausel im Zweck-Mittel-Prinzip analoge Parenthese im Kontext des Sittengesetzes bzw. des kategorischen Imperativs und damit die von Kant selbst (wenngleich eben nur parenthetisch) angedeutete Verknüpfung der beiden Imperativformen geht Seel jedoch ebenso wenig ein wie auf die im folgenden behandelten Konsequenzen, die diese Interpretation für das Begriffsverhältnis von Heteronomie und Autonomie hat. 
Der entscheidende Schritt von diesem analytischen Satz zum hypothetischen Imperativ besteht nun in der Ersetzung der Parenthese durch die entsprechende Aufforderungsformel:

If any rational agent wills the end, he ought to will the means.'

Der Grund für das Sollen der hypothetischen Imperative liegt demnach im möglichen Antagonismus zwischen unserem rationalen Wollen und einer davon abweichenden Neigung, welche letztere sich als handlungsleitend durchsetzen kann. Paton faßt dies folgendermaßen zusammen:

Perhaps it would be better to say, at least where the inclination prevails, that a rational volition is overcome by a less rational volition. It is the possibility of such defeat that gives rise to imperatives, and unless both types of volition were present in us, or at least possible in us, there would be no imperatives. ${ }^{24}$

Die Bedrohung unseres rationalen Wollens durch die Irrationalität dem entgegenstehender Neigungen, die sich dem rationalen Wollen und damit der Handlungsabsicht (gemäß der nach wie vor als richtig eingesehenen Zweck-Mittel-Relation) entgegenstellen können, ist der Grund für die Willensnötigung im Kontext instrumenteller Rationalität.

$\mathrm{Da}$ damit jedoch die Akten der instrumentellen Rationalität nicht bereits geschlossen sind, erkennt man nur, wenn man im Gegensatz zu Paton nicht der Auffassung ist, daß die hypothetischen Imperative im Kontext praktischer Philosophie letztlich bedeutungslos sind: „All he [sc. Kant] is doing is to clear them out of the way and so get on to the moral problem." (Paton 1947, 123) Paton kann sich mit dieser Bemerkung zwar auf die spätere Entwicklung Kants berufen, ja man kann gegenüber einer systematischen Aufwertung hypothetischer Imperative sogar geltend machen, daß Kant sich nur in der Grundlegung ausführlich mit ihnen beschäftigt hat. ${ }^{25}$

24 Ebd.; Cramer (1972, 198-199) verfehlt meines Erachtens bei seiner auch gegen Paton gerichteten Interpretation der hypothetischen Imperative das Phänomen der Willensschwäche, das genau dann vorliegt, wenn ein bereits gesetzter Zweck beibehalten wird, ohne jedoch die als notwendig und möglich erkannten Mittel zu dessen Erreichung zu ergreifen. Wir können - sozusagen wider besseres Wollen - einem konstanten Zweck zuwiderhandeln (s. im folgenden Korsgaards Beispiel der ,Prudence $)$. Eine willensschwache Person, deren Möglichkeit Kant mit der Aufstellung hypothetischer Imperative unterstellt, wird eben gerade nicht als eine, die ihre Zwecke ändert, adäquat beschrieben. Unter gewissen Umständen, daß nämlich die richtigen Mittel zur Zweckrealisierung erkannt wurden und auch in der Gewalt der betroffenen Person sind, kann das Ansinnen, „[...] aus dem Zustand des Wünschens in den des Wollens überzugehen " bzw. in bezug auf das Phänomen Willensschwäche eher aus dem Zustand des Wollens nicht in den des bloßen Wünschens überzugehen, sehr wohl die „Funktion eines hypothetischen Imperativs“ (Cramer 1972, 207) sein.

25 In der Kritik der praktischen Vernunft hat Kant die am Aufbau der Kritik der reinen Vernunft orientierte, wenngleich davon auch signifikant abweichende, moralphilosophische Gesamtkonzeption im Auge und konzentriert sich dementsprechend nicht auf hypothetische Imperative, sondern erstens auf das eine "Grundgesetz der reinen praktischen Vernunft " $\mathrm{KpV}$, AA 05: 30), zweitens auf den „Begriff [...] eines Gegenstandes der reinen praktischen Vernunft" (KpV, AA 05: 57), den Begriff des Guten also, und drittens auf die „Triebfedern der 
Doch Kant geht nach wie vor davon aus, daß Zweck-Mittel-Prinzipien normative Kraft besitzen, wenn er in der Kritik der Urteilskraft technisch-praktische Regeln von Naturgesetzen im eigentlichen Sinne der theoretischen Philosophie abgrenzt: „Doch heißen dergleichen praktische Regeln nicht Gesetze (etwa so wie physische), sondern nur Vorschriften: und zwar darum, weil der Wille nicht bloß unter dem Naturbegriffe, sondern auch unter dem Freiheitsbegriffe steht [...]." (KU, AA 05: 172; Hervorhebung: K.P.) Sobald wir Vorschriften für möglich und sinnvoll halten, haben wir den Bereich der Natur definitiv hinter uns gelassen.

Die Frage ist also, inwiefern hypothetische Imperative auch im Kontext praktischer Philosophie eine Relevanz besitzen, die über eine bloß technische und damit theoretische Diskussion hinausgeht. Dazu empfiehlt es sich, eine weitere prominente Interpretation dieses Kantischen Theoriestücks heranzuziehen.

\section{b) Christine M. Korsgaard}

Im Gegenzug zu Patons ausschließlich an Heteronomie orientierter Interpretation hypothetischer Imperative sieht Korsgaard in diesen bereits den Kontakt zur eigentlichen Kantischen Moralphilosophie und deren Zentrum, dem kategorischen Imperativ.

Korsgaard nähert sich der normativen Quelle instrumenteller Rationalität über eine Diskussion von praktischer Irrationalität bzw. Willensschwäche:

Timid Prudence says she has resolved to lead a more adventurous life, but when the opportunity for adventure knocks, Prudence always says ,tomorrow'. (Korsgaard 1997, 236)

Drei mögliche Erklärungen für Prudence' Verhalten sind nach Korsgaard nun denkbar:

(i) Prudence will eigentlich gar nicht unternehmungslustiger werden; mit ihrer ZweckAussage unterliegt sie entweder einer Selbsttäuschung oder sie belügt ihre Mitmenschen; von Willensschwäche würden wir in einem solchen Fall nicht sprechen.

reinen praktischen Vernunft“ (KpV, AA 05: 71), den Begriff der Achtung vor dem Gesetz. Und schließlich ist er in der KU der Auffassung, daß alle ,technisch-praktische Regeln [...] zur theoretischen Philosophie gezählt werden “ müssen (AA 05, 172; vgl. auch EEKU, AA 20: 195-201, insbes. die Anm. zu 200). Kant kehrt damit in dieser Hinsicht (und nur in dieser) zu seiner vorkritischen Auffassung zurück, vgl. UDG, AA 02: 298, sowie dazu Schwaiger 1999, 38-43. Die hypothetischen Imperative haben also das genuine Interesse Kants verloren, weil sie seiner neuen Ansicht nach nicht zum Kernbestand der praktischen Philosophie gehören, insofern sie die Richtigkeit der Handlung, d.h. die Klugheit oder Geschicklichkeit, nur gemessen an Standards der „Selbstliebe“ (KpV, AA 05: 22) betreffen. Vgl. dazu auch Carl Stange (1900, 236-240), der der Auffassung ist, Kant habe die hypothetischen Imperative im Lauf seiner Entwicklung zu Recht aus der Praktischen Philosophie in die Theoretische Philosophie verschoben, sowie im Gegenzug Cramer 1972, 196: „Es ist ein trivial zu nennender Sachverhalt, $\mathrm{da}[\mathrm{B}]$ eine technische oder pragmatische Vorschrift bezüglich dessen, was sie vorschreibt, als theoretische Aussage aufgefaßt werden muß. Dies bedeutet aber noch nicht, daß sie nicht als Vorschrift oder Gebot auftreten kann.“ 
(ii) Prudence will zwar zunächst unternehmungslustiger werden, geht jedoch von diesem Ziel ab, als sie bemerkt, welche Mittel sie dazu ergreifen müßte (inklusive solcher, die sie eventuell ins Gefängnis befördern könnten); auch in diesem Fall würden wir nicht von Willensschwäche sprechen, es liegt hier vielmehr eine möglicherweise vollkommen rationale Abwägung vor, wofür (in nicht-Kantischem Kontext) Bratman folgende treffende Charakterisierung gefunden hat: „One person's modus ponens is another's modus tollens. " 26

(iii) Prudence bleibt bei ihrem Vorsatz, auch unter Kenntnisnahme der nötigen Mittel; doch sobald sich die Gelegenheit eines Wagnisses bietet, wird sie von Furchtsamkeit, Trägheit oder Depression überwältigt, so daß sie das Ziel zwar weiterhin für erstrebenswert erachtet, sich jedoch schlicht außerstande sieht, entsprechend zu handeln; erst in diesem Szenario zeigt Prudence Willensschwäche, indem sie nicht so handelt, wie sie es selbst in dieser Situation für das Beste hält, und verletzt damit das Zweck-Mittel-Prinzip.

Dieses Fallbeispiel gibt Korsgaard nun die Möglichkeit, die Kantische Position mithilfe praktischer Syllogismen zu rekonstruieren:

Whoever wills the end wills the means.

I will the end.

$\rightarrow$ I will the means. (Korsgaard 1997, 238)

Die offenkundige Fehlerhaftigkeit dieses Syllogismus beruht auf der - wie Prudence' Verhalten gezeigt hat - fälschlich angenommenen Maior und führt Kant, so Korsgaards Interpretation, zum Einschalten der Rationalitätsklausel27:

26 Bratman 1987, 116. Neben diesen beiden Abweichungen von der geäußerten Handlungsabsicht, die sich mehr oder weniger auf den Zweck beziehen, könnte man sich auch eine solche vorstellen, die sich vorrangig auf die Mittel, genauer deren Fehleinschätzung, bezieht. Doch kann damit ebenfalls nicht das Phänomen der Willensschwäche geklärt werden, wie Staege dies nahelegt: „Die Unvernünftigkeit des Willens, die die Sofern-Klausel verständlich machen würde, könnte allenfalls darin gesucht werden, dass das als notwendig vorgestellte Mittel nicht-identisch ist mit dem (tatsächlich) notwendigen Mittel, der Wille seinen Zweck also irrtümlich unter Einsatz eines zweckwidrigen Mittels verfolgt." (Staege 2002, 44-45, Fn. 10) Ein defizitärer Wille (an den sich ein Imperativ richten kann) unterscheidet sich aber wesentlich von einer defizitären Erkenntnis. Korsgaard geht ihrerseits auf den Fall des Irrtums nicht ein, fordert für ihre Verhaltensdisjunktion aber auch keine Vollständigkeit.

27 Vgl. dazu bereits Cramer 1972, 164: „Da die in der ,Sofern'-Klausel genannte Bedingung, deren Erfüllung ein derartiges Gebot analytisch ausschließt, eben nicht immer erfüllt ist, muß jemandem, auf dessen Handlungen Vernunft [zu ergänzen: „möglicherweise“; K. P.] keinen entscheidenden Einfluß hat, das Ergreifen des Mittels geboten werden, das in seiner Gewalt ist. Dies hätte nur dann keinen Sinn, wenn der den Willen nicht näher qualifizierende Satz: ,Wer den Zweck will, will auch das unentbehrliche Mittel, das in seiner Gewalt ist' ein analytischer Satz wäre. Doch ist dieser Satz, so scheint es nun, nicht nur kein analytischer, sondern nicht einmal ein wahrer Satz. [...] Die Zusatzbedingung, die in die Protasis einzuführen ist, um den Gebotscharakter der Apodosis und damit die Nötigung des Willens verständlich zu machen, ist eingeführt, wenn die in der ,Sofern'-Klausel der Regel genannte Bedingung nicht erfüllt ist. Und für diese Möglichkeit scheint die erfahrungsmäßig nur zu oft konstatierbare Defizienz der Vernunft in Ansehung des Einsatzes rational einsichtiger Mittel für Zwecke, die man will, in der Tat einzustehen." Vgl. zur Analytizität des Zweck- 
Whoever wills the end wills the means in so far as he is rational.

I will the end.

$\rightarrow$ Therefore I will the means in so far as I am rational.

$\rightarrow$ Therefore I ought to will the means. ${ }^{28}$

Korsgaards zentrale These in diesem Kontext - „that there can be no instrumental norms unless there are also unconditional norms" 29 - rückt nun hypothetische Imperative zwar in eine scheinbar gefährliche Nähe zum kategorischen Imperativ. Es scheint die aus Kantischer Perspektive zu verhindernde Assimilation des kategorischen Imperativs an die hypothetischen Imperative zu drohen. Doch zum einen läßt sich diese Gefahr ausschließen, da die philosophische Bedeutung des Bestimmungsgrunds zu einer Handlung davon unberührt bleibt (Korsgaard geht darauf, soweit ich sehe, nicht ein) und zum anderen weist diese Strategie einen interessanten Weg zu der einzigen Quelle praktischer Normativität.

Die entscheidende Frage bezieht sich darauf, wie wir den zielgerichteten Willen konzipieren können, der weder per se die erforderlichen Mittel ergreift noch unabhängig von der Orientierung auf die Mittel verstanden werden kann: „The answer is that willing an end just is committing yourself to realizing the end. [...] To will an end is to give oneself a law, hence, to govern oneself. That law is not the instrumental principle; it is some law of the form: Realize this end." (Korsgaard 1997, 245) Korsgaard macht daran anschließend klar, inwiefern hypothetische Imperative tatsächlich in die praktische Philosophie gehören und selbst instrumentelle Rationalität nur in normativen Termini ausbuchstabiert werden kann:

It means that your willing the end gives it a normative status for you, that your willing the end in a sense makes it good. The instrumental principle can only be normative if we take ourselves to be capable of giving laws to ourselves - or, in Kant's own phrase, if we take our own wills to be legislative. [...] Who makes a law for whom? The answer in the case of the instrumental principle is that I make a law for me [sc. without universalizing it; K. P.]. And this is a law which I am capable of obeying or disobeying. ${ }^{30}$

Mittel-Prinzips auch Korsgaard: „The only proposition which Kant can claim is an analytic truth is the one with the caveat in it." (Korsgaard 1997, 238)

28 Ebd. 239. - Korsgaards weiterreichende Kritik dieses Syllogismus bezieht sich auf die scheinbar bloß den historischen Umständen geschuldete Hintergrundmetaphysik, die Kant dazu bewegt habe, dieses Sollen vom stets rationalen Wollen eines göttlichen oder heiligen Willens abzuleiten: „God does so-and-so (or, a perfectly rational being does so-and-so) and therefore I ought to do so-and-so." (Ebd.) Kant setzt zwar nicht Gott oder ein vollkommenes Wesen als Rechtsgrund des menschlichen Sollens voraus (schließlich betont er häufig, daß Religion auf Moral folgen, niemals aber dieser vorausgehen kann; vgl. z.B. KpV, AA 05: 40-41); beim „heiligen Willen“ (GMS, AA 04: 414) handelt es sich dementsprechend um eine Nominalbestimmung, deren objektive Realität in der Ethik kein argumentatives Gewicht trägt. Gleichwohl gilt ihm - und nicht nur ihm - die ontologische Prävalenz des Intelligiblen als unumstößlich. In bezug auf den Begriff der Normativität macht diese Prävalenz, die in der Deduktion des kategorischen Imperativs innerhalb der Grundlegung sogar dessen Rechtsgrund bezeichnet, jedoch nur das eine dessen beider Konstituentien aus. Die andere Seite der Medaille ist die Heteronomie.

29 Korsgaard 1997, 247, Fn. 65.

30 Ebd. 246. 
Wie ich im folgenden argumentieren werde, ist zwar die Universalisierung nach Kant nicht die einzige, vielleicht nicht einmal die entscheidende Differenz zwischen dem Feld moralischer und dem Feld technischer bzw. pragmatischer Handlungsmaximen, wie Korsgaard anzunehmen scheint. Vielmehr liegt die Differenz in den wesentlich unterschiedlichen Bestimmungsgründen $\mathrm{zu}$ einer Handlung (vgl. KpV, AA 05: $\mathbb{S}$ 2-6). Aber es ist Korsgaards Verdienst, die hypothetischen Imperative hinsichtlich ihrer normativen Kraft an den Begriff der Autonomie, wie Kant ihn im Kontext des kategorischen Imperativs diskutiert, herangeführt zu haben.

Aus Korsgaards Ausführungen wird jedoch letztlich nicht ganz klar, ob die Vernachlässigung einer irrationalen Anfechtung bei der Frage nach der normativen Kraft hypothetischer Imperative ein Charakteristikum der Kantischen Theorie selbst oder eine systematische Komplettierung durch Korsgaards entsprechendes eigenes Theorem sein soll (vgl. insbes. Korsgaard 1997, 240, Fn. 52). Wichtig scheint mir daher neben Korsgaards Betonung der Autonomie im Kontext hypothetischer Imperative auch eine deutliche Würdigung von Patons Herausarbeiten der Sinnlichkeit als Bedingung derer Autorität, und zwar im Sinne der Ursache einer drohenden Niederlage der Vernunft. Patons und Korsgaards Perspektiven auf die hypothetischen Imperative bezeichnen die beiden Seiten praktischer Normativität; Heteronomie und Autonomie kennzeichnen nach Kant, wie ich noch näher erläutern werde, den spezifisch gebotsoffenen Willen des Menschen.

III.

Ich habe die Analyse von Kants Konzept praktischer Normativität bis hierher anhand der hypothetischen Imperative durchgeführt; der entscheidende Zusatz, der appellative Rekurs auf die Rationalität des Menschen, der in Verbindung mit dem analytischen Zweck-Mittel-Prinzip zu einem Imperativ wird, ist hier sehr deutlich zu erkennen.

Es ist natürlich kein Zufall, daß Kant den soeben aus der Zweck-Mittel-Rationalität herausgeschälten Kern praktischer Normativität an unsere vernünftige (im Gegensatz zur bloß sinnlichen) Verfaßtheit knüpft. Doch wird dies noch greifbarer, wenn man sich die Medaille der Willensbestimmung von der anderen Seite, dem kategorischen Imperativ her ansieht. Nachdem ich den kategorischen Imperativ also zunächst ausgeklammert habe, weil Abwägung und damit instrumentelle Rationalität in ihm keine Rolle spielen, zeigt sich nun, daß seine Berücksichtigung in diesem Kontext genau dann lohnt, wenn man gesehen hat, daß sich selbst hinter dem Konzept instrumenteller Rationalität bei Kant eine irreduzibel normative Dimension eröffnet. Im folgenden möchte ich daher statt der Hauptdifferenz zwischen möglichen Imperativen ihre gemeinsame Wurzel behandeln.

Vorab nur kurz und thesenartig die Tendenz meiner Interpretation: Erstens, daß aus dem Zweck-Mittel-Prinzip eine Nötigung des Willens wird, erfordert, wie im 
vorstehenden ausgeführt, einen Rekurs auf den Einfluß der Vernunft auf unsere Handlung. Zweitens, daß aus dem Sittengesetz, das für den gesamten mundus intelligibilis, also für alle vernünftigen Wesen gilt, ein kategorischer Imperativ und damit ebenfalls eine Nötigung des Willens wird, erfordert einen (negativen) Rekurs auf unsere Endlichkeit bzw. Sinnlichkeit. Drittens, die Quelle der Normativität liegt allein in unserer spezifisch menschlichen Rationalität ${ }^{31}$, die von der Seite hypothetischer Imperative aus betrachtet eine Übersteigung bloßen Verhaltens hin zu einem absichtsvollen und begründeten Handeln ermöglicht, und von der Seite des kategorischen Imperativs aus betrachtet ein mögliches Unterlaufen absoluter Sittlichkeit durch sinnliche Affektion erzwingt. Daß diese Rationalität eine einzige ist, dafür spricht nicht nur das Kantische Ideal der Einheit der Vernunft, sondern auch seine konkrete Konzeption von Normativität. Es wäre mindestens verwunderlich, wenn Kant für verschiedene Imperativformen unterschiedliche Quellen praktischer Normativität anschlagen würde.

Im einzelnen: praktische Normativität bleibt bei Kant natürlich nicht auf hypothetische Imperative beschränkt; die dort gefundene Rationalitätsklausel, die sich für den imperativen Charakter der hypothetischen Imperative verantwortlich zeigte, sollte sich mutatis mutandis auch am kategorischen Imperativ wiederfinden (das bedeutet oder begründet nicht, daß eine der beiden Imperativformen - wie auch immer - auf die andere reduzierbar oder aus dieser abzuleiten wäre; eine solche These vertrete ich ausdrücklich nicht). Wohl gibt es den Unterschied zwischen instrumenteller, prudentieller und moralischer Rationalität. Doch die Tatsache, daß Kant auch die ersten beiden, also instrumentelle und prudentielle Rationalität, in normativen Termini behandelt und insofern von hypothetischen Imperativen spricht, rückt auch diese Formen der Rationalität aus dem Bereich psychologischer Deskription in eine normative Sphäre. Und innerhalb dieser Sphäre werden Imperative nur dahingehend unterschieden, daß sie entweder material bestimmt sind, d.h. daß der Bestimmungsgrund zu einer Handlung im Erreichen eines Zwecks durch die Ergreifung als adäquat erkannter Mittel liegt, oder formal bestimmt sind, d.h. daß der Bestimmungsgrund zu einer Handlung in der Anerkennung sittlicher Gesetzmäßigkeit liegt. Genau diese Varianz der Bestimmungsgründe möglicher Handlungen markiert die differentia specifica möglicher Imperative.

Kant geht sowohl in der Grundlegung als auch in der Kritik der praktischen Vernunft davon aus, daß Wesen, die ihre Neigungen unter ständiger rationaler Kontrolle hätten, stets sittlich handeln würden. Er führt in diesem Kontext nominal als Kontrast zum menschlichen Willen den Begriff bzw. die Idee des ,heiligen Willen[s]“ ein, „d. i. einen solchen, der keiner dem moralischen Gesetze widerstreitenden Maximen fähig wäre“ “. ${ }^{32}$ Analog zu materiellen Körpern, die ausnahmslos Naturgesetze befolgen, gehorchten diese rein geistigen Vernunftwesen stets dem Gesetz

31 Vgl. zu diesem Begriff oben Fn. 4.

$32 \mathrm{KpV}$, AA 05: 32; vgl. zum heiligen Willen bereits die GMS, z.B. AA 04: 414, 439. 
der sittlichen Welt. Die Allgemeinheit und Notwendigkeit dieser Gesetze dulden keine Ausnahmen. Das Prinzip „in mundo non datur casus“ (KrV, B 280) darf man im Sinne Kants getrost von der sensiblen auf die intelligible Welt übertragen. Wesen, die lediglich Bürger dieser intelligiblen Welt sind, handeln ohne Ausnahme sittengesetzlich. Für Wesen mit einem solchen absolut-guten Willen fällt daher das objektive mit dem subjektiven Prinzip des Wollens zusammen:

[...] das objective Princip [sc. des Wollens; K. P.] (d. i. dasjenige, was allen vernünftigen Wesen auch subjectiv zum praktischen Princip dienen würde, wenn Vernunft volle Gewalt über das Begehrungsvermögen hätte) ist das praktische Gesetz. (GMS, AA 04: 400)

Auf die von mir hervorgehobene Kautel werde ich sogleich genauer eingehen. -

Ein Imperativ, also eine Aufforderung, diesem Gesetz zu gehorchen, wäre jedenfalls bei so konstruierten Heiligen überflüssig eben deswegen, weil ein alternatives, d.h. gesetzwidriges Verhalten diesen Wesen aufgrund der absolut-rationalen Beherrschung ihrer Sinnlichkeit gar nicht zur Disposition stünde (bezogen auf ihr Wollen, nicht auf ihr Können). Daß jedoch ein Imperativ bei Heiligen wertlos wäre, bedeutet nicht, daß das Sittengesetz für sie deskriptiven Charakter hätte; ,von außen' betrachtet mag das zwar so aussehen, doch besitzt es für diese (problematischen) autonomen Wesen präskriptiven Charakter und muß damit vom Naturgesetz unterschieden werden. Im Gegensatz zum fallenden Stein folgten auch Heilige wie alle vernünftige Wesen - einem Gesetz nur, weil sie dieses anerkennen; die Assimilierung eines Heiligen an einen fallenden Stein wäre mit Sicherheit nicht im Sinne Kants. ${ }^{33}$

Bei Vernunftwesen, die nicht ausschließlich sich selbst bestimmen, sondern auch von Neigungen bestimmt werden können, also (beispielsweise) dem Menschen als einem von „Bedürfnissen und sinnlichen Bewegursachen afficirte[n] Wesen“ (KpV, AA 05: 32), verhält sich die Sache hingegen anders als bei reinen Vernunftwesen: „Ist es [das praktische Gesetz; K. P.] nicht zugleich subjectiv immer kräftig gnug zur Handlung so heißt die Nothwendigkeit Verbindlichkeit die Handlung selbst Pflicht und die Formel ein absolutes sollen." (HN, AA 23: 245) Wir können dem Sittengesetz gemäß handeln, aber wir können auch ihm zuwider handeln. Wir können uns in unseren Handlungen vom Anspruch der Moralität bestimmen lassen, wir können uns diesem Anspruch aber auch verschließen und uns stattdessen von unseren Neigungen bestimmen lassen. Da wir nicht per se sittlich handeln, nimmt das Sittengesetz - also „nur dasjenige zu wählen, was die Vernunft unabhängig von der Neigung als praktisch nothwendig [...] erkennt" (GMS, AA 04: 412) - für uns Bürger zweier Welten die „Form eines Imperativs“ (KpV, AA 05: 32) an. Nur für endliche Wesen wie uns Menschen ist der „Begriff der Pflicht“ relevant, „, der den eines guten Wil-

33 Vgl. dazu KpV, AA 05: 32: „Dieses Princip der Sittlichkeit nun [...] erklärt die Vernunft zugleich zu einem Gesetze für alle vernünftige Wesen, so fern sie überhaupt einen Willen, d. i. ein Vermögen haben, ihre Causalität durch die Vorstellung von Regeln zu bestimmen [...].“ (Hervorhebung: K. P.) Vgl. dazu auch Wood 1999, 174 und 379, sowie Anderson 2001, 277. 
lens, obzwar unter gewissen subjectiven Einschränkungen und Hindernissen, enthält". ${ }^{34}$ Wir Menschen müssen uns als von Heteronomie umlagerte Autonomie verstehen, weil wir einerseits von Neigungen, d.h. nicht-vernünftigen Beweggründen, affiziert werden, uns aber andererseits diesen gegenüber vernünftig verhalten können, d.h. wir können die sittliche Forderung gegenüber sinnlichen Neigungen vorziehen, ohne daß dadurch allerdings unsere Sinnlichkeit als solche verschwinden würde.

Der kategorische Imperativ richtet sich demnach an denjenigen Willen, dessen subjektiver Bestimmungsgrund, das principium executionis, möglicherweise vom objektiven, d.h. sittlichen, Bestimmungsgrund, dem principium dijudicationis abweicht; aus der Perspektive allgemeiner moralischer Gesetzmäßigkeit der Handlungen sollen diese beiden Prinzipien in Konkordanz gebracht werden. Das für uns geltende moralische Sollen hängt damit wesentlich davon ab, daß bei endlichen Wesen die Vernunft möglicherweise nicht die, volle Gewalt über das Begehrungsvermögen` hat.

In diesem Sinne bestimmt Kant den kategorischen Imperativ als einen „synthetisch-praktische[n] Satz a priori“ (GMS, AA 04: 420), weil durch ihn das Wollen einer bestimmten Handlung „mit dem Begriffe des Willens eines vernünftigen Wesens unmittelbar als etwas, das in ihm nicht enthalten ist, verknüpft“ (ebd.) wird. Gerade diese Synthetizität des kategorischen Imperativs, d.h. die bei endlichen Wesen wie dem Menschen mögliche formale Differenz zwischen einer Maxime und dem Sittengesetz, ist für den imperativen Charakter des Sittengesetzes für endliche Wesen verantwortlich.

Der kategorische Imperativ, d.h. der Aufruf zur Subsumtion subjektiver Handlungsgrundsätze unter das Sittengesetz, fordert damit die Willensbestimmung durch das allgemeine Gesetz - jedoch nicht unmittelbar, sondern im „Streit [...], den jetzt die moralische Gesinnung mit den Neigungen zu führen hat" (KpV, AA 05: 147). Während ein heiliger Wille bereits als solcher die Verknüpfung des Willens mit der moralischen Handlung vollziehen würde, unterlassen wir Menschen diese möglicherweise aufgrund unserer subjektiv-sinnlichen ,Befangenheit‘:

34 GMS, AA 04: 397; Hervorhebung: K. P.; vgl. auch GMS, AA 04: 439 zur weiteren Begriffsklärung von Pflicht und Verbindlichkeit bezüglich der Differenz von heiligem und nichtheiligem Willen, sowie zu Kants Differenzierung des heiligen und des menschlichen Willens hinsichtlich der in Frage stehenden Normativität auch GMS, AA 04: 414. Von der nicht weniger deutlichen Differenzierung in GMS, AA 04: 412-413 betont Korsgaard zu Recht, daß Kant sie vor der weiteren Differenzierung der Imperative in hypothetische und den kategorischen vornimmt: „[...] imperatives are addressed to beings who may follow them or not. And this is true of the instrumental principle as well as of the others." (Korsgaard 1997, 236) Unmißverständlich heißt es auch in der etwa parallel zur Grundlegung enstandenen Moral Mrongovius II: „Die Imperative sind aus einer Idee eines vollkommenen Willens hergenommen und gelten als Regeln für meinen unvollkommenen Willen; Pflicht ist eine Idee eines vollkommenen als eine norm eines unvollkommenen Willens." (VMron, AA 29: 606) 
Ich verknüpfe mit dem Willen ohne vorausgesetzte Bedingung aus irgend einer Neigung die That a priori, mithin nothwendig (obgleich nur objectiv, d. i. unter der Idee einer Vernunft, die über alle subjective Bewegursachen völlige Gewalt hätte). ${ }^{35}$

Die parenthetisch formulierte Einschränkung bezüglich der „Idee einer Vernunft, die über alle subjective Bewegursachen völlige Gewalt hätte" bezeichnet hier dasselbe wie die bereits angeführte Klausel in Kants Differenzierung zwischen subjektivem und objektivem Prinzip des Wollens („wenn Vernunft volle Gewalt über das Begehrungsvermögen hätte“; GMS, AA 04: 400).

Auffällig ist nun hierbei, daß dieser Vorbehalt analog demjenigen zu verstehen ist, der sich im Kontext der hypothetischen Imperative in den Bestimmungen der Zweck-Mittel-Relation ebenfalls als Parenthese fand. Hier wie dort stammt die nötigende Kraft aus der eingeschobenen Klausel, die verantwortlich ist für die Umwandlung von Behauptungen in Imperative.

Mit einer Gegenüberstellung der entsprechenden Vorbehalte läßt sich diese Explikation des Normativen durch den Appell an unsere spezifisch menschliche, d.h. endliche Rationalität verdeutlichen:

War der Übergang vom analytischen Zweck-Mittel-Prinzip zum hypothetischen Imperativ in jenem Zusatz zu lokalisieren:

so fern die Vernunft auf seine Handlungen entscheidenden Einfluß hat (GMS, AA 04: 417),

so findet sich der Übergang vom Sittengesetz zum kategorischen Imperativ in diesem:

wenn Vernunft volle Gewalt über das Begehrungsvermögen hätte (GMS, AA 04: 400).

Das in diesen beiden Klauseln benannte Desiderat steht damit hinter den beiden möglichen Imperativformen. Was sich im Fall hypothetischer Imperative als Willensschwäche in bezug auf den als richtig erkannten Mitteleinsatz zu einem selbstgesetzten Zweck äußern kann, kann sich im Fall des kategorischen Imperativs als Willensschwäche ${ }^{36}$ in bezug auf eine als sittlich erachtete Handlung äußern. Die Differenz der Imperative bezieht sich also, wie bereits bemerkt, auf die unterschiedlichen Bestimmungsgründe zu einer Handlung: während hypothetische Imperative die Normativität an einen willkürlich gesetzten Zweck binden, liegt das moralische principium executionis in der „Achtung fürs moralische Gesetz“37, einer Selbstachtung der Vernunft, der „Achtung für sein eigenes Wesen“38. Die gemeinsame Wurzel

35 GMS, AA 04: 420 Anm.; Hervorhebung: K. P.; Kant spricht in dieser Anmerkung vom kategorischen Imperativ und dem Sittengesetz promiscue, was lediglich als sprachliche Ungenauigkeit gewertet werden kann, da er im angeführten Kontext eines göttlichen bzw. heiligen Willens eben diese Unterscheidung einführt.

36 Vgl. zu Kants Differenzierung der Gründe für Handlungen, die sich nicht am Sittengesetz orientieren auch RGV, AA 06: 28-32.

$37 \mathrm{KpV}$, AA 05: 73; vgl. auch GMS, AA 04: 400.

38 MS, AA 06: 403; vgl. zum Gefühl der Achtung, das „nicht zu Beurtheilung der Handlungen, oder wohl gar zur Gründung des objectiven Sittengesetzes selbst [dient], sondern blos zur Triebfeder, um dieses in sich zur Maxime zu machen “ (KpV, AA 05: 76), auch Pollok 2006. 
aller Imperative, die Bedingung der Möglichkeit jeglichen Sollens, liegt hingegen in der praktischen Vernunft des Adressaten. Was im Fall hypothetischer Imperative mit dem Verweis auf den selbstgesetzten Zweck (und die zu dessen Erreichung als erforderlich erkannten Mittel) begründet wird, findet im Fall des kategorischen Imperativs die Begründung in einer möglichen allgemeinen Gesetzgebung, d.h. die Vernünftigkeit der Handlungen besteht in dem von Kant festgesetzten Wert des Intelligiblen selbst. Eine weitere Frage „wozu? “ wäre für Kant in diesem Kontext abwegig - lapidar heißt es bereits in der Grundlegung: „die vernünftige Natur existirt als Zweck an sich selbst.“ (GMS, AA 04: 429) Und im Zusammenhang der „Deduction des obersten Princips der Moralität" (GMS, AA 04: 463) erklärt Kant:

Weil aber die Verstandeswelt den Grund der Sinnenwelt, mithin auch der Gesetze derselben enthält, $[\ldots]$ so werde ich mich als Intelligenz, obgleich andererseits wie ein zur Sinnenwelt gehöriges Wesen, dennoch dem Gesetze der ersteren, d. i. der Vernunft, die in der Idee der Freiheit das Gesetz derselben enthält, und also der Autonomie des Willens unterworfen erkennen [...]. (GMS, AA 04: 453-454)

Der Indikativ des nun mehrfach genannten Klammerausdrucks im instrumentellen Kontext (,so fern die Vernunft auf seine Handlungen entscheidenden Einfluß hat"; GMS, AA 04: 417) erhebt uns also aus der Sphäre bloß instinktiven Verhaltens in die Sphäre praktischer Normativität und schafft damit einen Übergang vom naturalen Geschehen zur Verbindlichkeit rationaler Konsequenz ${ }^{39}$; der Konjunktiv des Klammerausdrucks im moralischen Kontext (,,wenn Vernunft volle Gewalt über das Begehrungsvermögen hätte “; GMS, AA 04: 400) hingegen bezeichnet gerade die obere Grenze dieser Sphäre, die zu verlassen uns endlichen Wesen eben unmöglich ist. Denn die Tatsache, daß wir keinen absolut-guten Willen besitzen, objektives und subjektives Prinzip des Wollens bei uns also nicht per se identisch sind, markiert hier den Übergang vom Praktischen Gesetz zum kategorischen Imperativ. Die Sphäre praktischer Normativität ist damit einerseits begrenzt von vernunftlosem Verhalten und andererseits von der „praktische[n] Idee“ einer „Heiligkeit des Willens“ (KpV, AA 05: 32). Exakt zwischen diesen beiden Grenzen, zum bloß Natürlichen einerseits und zum Göttlichen andererseits, befindet sich die Sphäre menschlicher Rationalität, die Kant im Kontext Praktischer Philosophie durch das ausschließlich hier zu findende Sollen kennzeichnet. ${ }^{40}$

39 Im Sinne eines solchen Übergangs spricht Kant vom Menschen auch von einem Tier, „welches Disciplin nöthig hat" (Anthropologie-Parow von 1772/73, VParow, AA 25/1: 425, vgl. auch Reflexion 1423, AA 15: 621). Vgl. auch Kants Definition eines vorbegrifflichen Begehrungsvermögens in den Vorarbeiten zur Tugendlehre: „Naturtrieb (instinct) ist ein Bestimmungsgrund des Begehrens von etwas vor aller Kenntnis des Gegenstandes (Durst Hunger Geschlechtstrieb)“ (HN, AA 23: 378), sowie im Gegensatz zum Instinkt Kants Definition des Willens als eines „Begehrungsvermögen[s], sofern es nur durch Begriffe, d.i. der Vorstellung eines Zwecks gemäß zu handeln, bestimmbar ist“ (KU, AA 05: 220; vgl. auch KpV, AA 05: 9 sowie MS, AA 06: 211).

40 Zur Weiterbestimmung dieser Normativität müßte man im Sinne Kants selbstverständlich die „Kategorien der Freiheit“ (KpV: AA 05: 66) berücksichtigen; im Rahmen dieses Aufsatzes muß auf eine ausführliche Erörterung jedoch verzichtet werden. Eine Verbindung 
Jenseits der für Kant zweifellos gewichtigen Differenz zwischen hypothetischen Imperativen und dem kategorischen Imperativ bezüglich der heteronomen bzw. autonomen Bestimmungsgründe zu einer Handlung gründen beide Formen in dem Kantischen Axiom, daß der Begriff des Sollens den Begriff der Vernunft voraussetzt (ausdrücklich nicht mit diesem identisch ist, wie Kants nominale Einführung des heiligen Willens zeigt).

Die „praktische [...] Bestimmung des Menschen“ (KpV, AA 05: 146) - seine Auszeichnung gegenüber absoluter Sinnlichkeit und absoluter Vernünftigkeit sowie seine Aufgabe der „Beförderung des höchsten Guts“ (KpV, AA 05: 114) - gründet

zwischen der jeweiligen normativen Kraft möglicher Imperative und diesen Kategorien der Freiheit stellt Susanne Bobzien her: „Wie durch die Kategorien der Natur das Mannigfaltige der sinnlichen Anschauungen unter die Einheit des apriorischen Bewußtseins, ich denke' gebracht wird, so wird [...] durch die Kategorien der praktischen Vernunft das Mannigfaltige der Begehrungen unter die Einheit des apriorischen Bewußtseins, ich soll (tun)' bzw. ,ich will als vernünftig (tun)` gebracht, wobei dieses Bewußtsein in der ersten Formulierung unter dem Aspekt, einer im moralischen Gesetze gebietenden Vernunft', in der zweiten Formulierung unter dem Aspekt, eines reinen Willens a priori ${ }^{6}(\mathrm{KpV}, \mathrm{AA} 05$ : 65.24-26) gesehen wird.“ (Bobzien 1988, 197) Alle absichtsvollen Handlungen, also all dasjenige, was entweder Gegenstand eines hypothetischen oder des kategorischen Imperativs sein kann, kann aus der Perspektive eines Sollens bzw. eines vernünftigen Wollens betrachtet werden. Bobzien ist nun der Auffassung, daß nach Kant für moralische Handlungen im engeren Sinne auch Modalitätskategorien erfordert werden, während für $\mathrm{Ge}$ genstände der instrumentellen bzw. prudentiellen Rationalität die ersten drei Kategorientitel der Quantität, Qualität und Relation ausreichen: „In diesem Falle bleibt den Kategorien der Gruppen 1 bis 3 die Funktion, Handlungen als Wirkungen von Zwecksetzungen zu erklären. Das heißt, die Kategorien dienen in diesem Falle nicht dazu, eine Handlung als praktische geltungsdifferent zu bestimmen, sondern dazu, eine Antwort auf die (theoretische) Frage zu ermöglichen, warum hat dieses oder jenes ,Naturereignis Handlung stattgefunden? Hierzu liefern die Kategorien den Begriff des Wohls [...] sowie den Begriff des Nützlichen [...].“ (Ebd. 209) Doch Bobziens Aufteilung der Kategoriengruppen und damit ihre Interpretation der praktischen Kategorien insgesamt führen in eine Schwierigkeit hinsichtlich der objektiven Realität instrumenteller Vernunft: „Der von Kant im Kategorienabschnitt durchweg betonte Bezug der Kategorien der praktischen Vernunft auf die freie Willkür, wie auch schon die Benennung dieser Kategorien als ,Kategorien der Freiheit' zeigen, daß Kant hier nur die (moralisch-)praktische Funktion der Kategorien behandelt - und auch nur für diese Funktion der Kategorien wurde die objektive Realität bewiesen. Andrerseits muß eine theoretische Erklärung der Handlungen als Erscheinungen stets prinzipiell auch möglich sein, damit der Kausalzusammenhang innerhalb der ,Erscheinungswelt' nicht durchbrochen wird.“ (Ebd.) Die Konsequenz aus dieser Lektüre ist aber, daß die ersten drei Kategoriengruppen keine objektive Realität haben, solange sie nicht auf moralische, d.h. gute/böse, Handlungen angewandt werden. Damit haben sie aber die von Bobzien veranschlagte „Nebenfunktion [...] als theoretische oder technisch-praktische Begriffe" (ebd.) gerade nicht, denn ihre diesbezügliche Geltung bedürfte einer eigenen transzendentalen Deduktion. Letzteres wird von Bobzien nicht in Erwägung gezogen. Handlungen, deren technisch-praktische Betrachtung nicht bloß ein Durchgangsstadium zur moralisch-geltungsvarianten Betrachtung darstellt, also alle Handlungen der instrumentellen bzw. prudentiellen Rationalität könnten nach Bobziens Interpretation nicht einmal im vollen Wortsinne Handlungen sein, weil die dazu erforderliche objektive Realität der Kategoriengruppen 1 bis 3 nicht gesichert ist. 
im Appell an seine vernünftige Willensbestimmung - im Fall hypothetischer Imperative das Wollen der als adäquat erkannten Mittel zum handlungseffektiven Wollen zu machen, im Fall des kategorischen Imperativs das subjektive Prinzip des Wollens in Einklang zu bringen mit dem objektiven Prinzip des Wollens. Dieser Appell - „gestatte der Vernunft entscheidenden Einfluß auf deine Handlung!“ - ist möglich, weil der Mensch sich kraft seiner Autonomie über seine sinnlichen Affektionen hinwegsetzen kann und insofern ein Glied der Verstandeswelt ist; dieser Appell ist aber zugleich auch nötig, weil der Mensch nicht naturgemäß richtig handelt, sondern durch Heteronomie ,bedroht ${ }^{`}$ ist, wodurch er zu einem Glied der Sinnenwelt wird.

Der Mensch ist Kant zufolge also (i) ein der Überlegung fähiges, also rationales Wesen, (ii) ein heteronomes und (iii) zugleich autonomes Wesen. Erst die Synthese der letzten beiden Aspekte - Heteronomie und Autonomie vereint - ergibt den Begriff des Sollens. ${ }^{41}$

\section{Literatur}

Anderson, R. Lanier (2001): Synthesis, Cognitive Normativity, and the Meaning of Kant's Question, ,How are synthetic cognitions a priori possible?' In: European Journal of Philosophy 9, 275-305.

Bobzien, Susanne (1988): Die Kategorien der Freiheit bei Kant. In: Kant. AnalysenProbleme - Kritik. Hrsg. von Hariolf Oberer und Gerhard Seel. Würzburg, 193-220.

Bratman, Michael E. (1987): Intentions, Plans, and Practical Reason. Cambridge/ Mass.

Broome, John (Manuskript 2005): Reasoning.

Cramer, Konrad (1972): Hypothetische Imperative? In: Rehabilitierung der praktischen Philosophie, Bd. I. Hrsg. von Manfred Riedel. Freiburg, 159-212.

Forst, Rainer (1999): Praktische Vernunft und rechtfertigende Gründe. Zur Begründung der Moral. In: Motive, Gründe, Zwecke. Theorien praktischer Rationalität. Hrsg. von Stefan Gosepath. Frankfurt/M., 168-205.

- (2004): Moralische Autonomie und Autonomie der Moral. Zu einer Theorie der Normativität nach Kant. In: Deutsche Zeitschrift für Philosophie 52/2, 179-197.

41 Diese Rekonstruktion ermöglicht unabhängig von der Frage nach dem Status der verschiedenen Imperative in Kants Spätphilosophie (vgl. oben Fn. 25) eine Identifikation seiner kritischen Moralphilosophie mit späteren Ausführungen, insofern die drei eben angeführten Elemente Heteronomie, Rationalität und Autonomie mit den Elementen der Bestimmung des Menschen korrelieren, die Kant in der Religionsschrift als „Tierheit“, „Menschheit“ und „Persönlichkeit“ bezeichnet (RGV: AA 06: 26); vgl. dazu auch oben Fn. 4. In der Anthropologie in pragmatischer Hinsicht von 1798 schließlich erweitert Kant die Bestimmung des Menschen noch um den Aspekt der Gesellschaft bzw. der Gattung (vgl. Anth, AA 07: 324-330). 
Habermas, Jürgen (1991): Vom pragmatischen, ethischen und moralischen Gebrauch der praktischen Vernunft. In ders.: Erläuterungen der Diskursethik. Frankfurt/M., 100-118.

Henrich, Dieter (1960): Der Begriff der sittlichen Einsicht und Kants Lehre vom Faktum der Vernunft. In: Die Gegenwart der Griechen im neueren Denken. Hrsg. von Dieter Henrich. Tübingen, 77-115.

Hill, Thomas E., Jr. (1973): The Hypothetical Imperative. In: Philosophical Review $82,429-450$.

Kain, Patrick (2003): Prudential Reason in Kant's Anthropology. In: Essays on Kant's Anthropology. Hrsg. von Brian Jacobs und Patrick Kain. Cambridge, 230-265.

Korsgaard, Christine (1996): The Sources of Normativity. Cambridge.

- (1997): The Normativity of Instrumental Reason. In: Ethics and Practical Reason. Hrsg. von Garrett Cullity und Berys Gaut. Oxford, 215-254.

Ludwig, Bernd (1999): Warum es keine, hypothetischen Imperative“ gibt, und warum Kants hypothetisch-gebietende Imperative keine analytischen Sätze sind. In: Aufklärung und Interpretation. Studien zu Kants Philosophie und ihrem Umkreis. Hrsg. von Heiner F. Klemme, Bernd Ludwig, Michael Pauen und Werner Stark. Würzburg, 105-124.

Paton, Herbert J. (1947): The Categorical Imperative. A Study in Kant's Moral Philosophy. London.

Patzig, Günther (1966): Die logischen Formen praktischer Sätze in Kants Ethik. In: Kant-Studien 56, 237-252.

Pollok, Konstantin (2006): Kant und Habermas über das principium executionis moralischer Handlungen. In: Moralische Motivation. Kant und die Alternativen. Hrsg. von Heiner F. Klemme, Manfred Kühn und Dieter Schönecker. Hamburg, 193-227.

Reinhold, Carl Leonhard (1791): Ueber das Fundament des philosophischen Wissens. Jena.

Schönecker, Dieter: Kant: Grundlegung III. Die Deduktion des kategorischen Imperativs. Freiburg/München 1999.

- / Wood, Allen W. (2002): Kants „Grundlegung zur Metaphysik der Sitten“. Ein einfübrender Kommentar. Paderborn.

Schwaiger, Clemens (1999): Kategorische und andere Imperative. Zur Entwicklung von Kants praktischer Philosophie bis 1785. Stuttgart.

Seel, Gerhard: Sind hypothetische Imperative analytische praktische Sätze? In: Grundlegung zur Metaphysik der Sitten. Ein kooperativer Kommentar. Hrsg. von Otfried Höffe. Frankfurt/M. 1989, 148-171.

Staege, Roswitha (2002): Hypothetische Imperative. In: Kant-Studien 93, 42-56.

Stange, Carl: Der Begriff der ,hypothetischen Imperative“ in der Ethik Kants. In: Kant-Studien 4, 1900, 232-247.

Steigleder, Klaus (2002): Kants Moralphilosophie. Die Selbstbezüglichkeit reiner praktischer Vernunft. Stuttgart. 
Willaschek, Marcus (1992): Praktische Vernunft. Handlungstheorie und Moralbegründung bei Kant. Stuttgart.

Wood, Allen W. (1999): Kant's Ethical Thought. Cambridge.

- / Schönecker, Dieter (2002): Kants „Grundlegung zur Metaphysik der Sitten“. Ein einführender Kommentar. Paderborn. 